\title{
Vorticity, Kinetic Energy, and Suppressed Gravitational-Wave Production in Strong First-Order Phase Transitions
}

\author{
Daniel Cutting $\odot,{ }^{1,2, *}$ Mark Hindmarsh $\odot,{ }^{1,2, \dagger}$ and David J. Weir $\oplus^{2,3, *}$ \\ ${ }^{1}$ Department of Physics and Astronomy, University of Sussex, Falmer, Brighton BN1 9QH, United Kingdom \\ ${ }^{2}$ Department of Physics and Helsinki Institute of Physics, PL 64, FI-00014, University of Helsinki, Finland \\ ${ }^{3}$ School of Physics and Astronomy, University of Nottingham, Nottingham NG7 2RD, United Kingdom
}

(Received 27 June 2019; revised 26 November 2019; accepted 26 June 2020; published 10 July 2020)

\begin{abstract}
We have performed the first three-dimensional simulations of strong first-order thermal phase transitions in the early universe. For deflagrations, we find that the rotational component of the fluid velocity increases as the transition strength is increased. For detonations, however, the rotational velocity component remains constant and small. We also find that the efficiency with which kinetic energy is transferred to the fluid falls below theoretical expectations as we increase the transition strength. The probable origin of the kinetic energy deficit is the formation of reheated droplets of the metastable phase during the collision, slowing the bubble walls. The rate of increase in the gravitational wave energy density for deflagrations in strong transitions is suppressed compared to that predicted in earlier work. This is largely accounted for by the reduction in kinetic energy. Current modeling therefore substantially overestimates the gravitational wave signal for strong transitions with deflagrations, in the most extreme case by a factor of $10^{3}$. Detonations are less affected.
\end{abstract}

DOI: $10.1103 /$ PhysRevLett.125.021302

The Laser Interferometer Space Antenna (LISA), scheduled for launch in 2034, will open the $\mathrm{mHz}$ band of the emerging field of gravitational wave astronomy [1]. One of the most exciting goals of LISA is to probe the early universe by searching for gravitational wave signals from a first-order phase transition.

While the standard model is a crossover [2,3], there are many extensions with first-order phase transitions. These range from adding a scalar singlet [4-8] or doublet [9-11], to models with spontaneously broken conformal symmetry [12-18]. There are also models with phase transitions in hidden sectors [19-25]. Nonperturbative methods are sometimes necessary to establish the order of the phase transition [26-28].

An important parameter of a first-order phase transition is the trace anomaly difference, which quantifies the energy available for conversion to shear stress, and hence the power of the gravitational wave signal. If the trace anomaly difference is comparable to the radiation energy density of the universe, we call the transition "strong". We denote the ratio of the trace anomaly to the thermal energy $\alpha$, in which case a strong transition has $\alpha \sim 1$. We call $\alpha \gg 1$ "very strong"; our results do not access this region.

Published by the American Physical Society under the terms of the Creative Commons Attribution 4.0 International license. Further distribution of this work must maintain attribution to the author(s) and the published article's title, journal citation, and DOI. Funded by SCOAP .
Substantial progress has been made in understanding gravitational wave production from first-order transitions with weak $\left(\alpha \sim 10^{-2}\right)$ to intermediate $\left(\alpha \sim 10^{-1}\right)$ strength using numerical simulations [29-32], as well as modeling [33-35]. While the fluid motion is well described as a linear superposition of sound waves after a weak transition [29], rotational modes and turbulence are expected in stronger transitions [36,37], which could substantially affect the gravitational wave signal [38-43].

At the same time, investigation of the underlying particle physics models indicates that intermediate to strong transitions are common in conservative extensions of the Standard Model $[44,45]$, and very strong transitions are possible in models of composite Higgs and nearly conformal potentials [12-18]. It is also clear that LISA will be most likely to observe transitions where nonlinear effects like shocks and turbulence become important [32]. Recent work tackling the nonlinear regime includes gravitational wave production from magnetohydrodynamic turbulence [46] and studies of shock collisions using a mixture of onedimensional simulations and modeling [47].

In this paper, we present results from the first numerical simulations of strong first-order phase transitions. We measure the fraction of the fluid kinetic energy in rotational modes, as traced by the mean-square velocity. As we increase the strength of the transition, this proportion grows substantially for deflagrations, with up to $65 \%$ of the mean square velocity found in rotational motion. The rotational proportion is far less for detonations, remaining roughly constant for all transition strengths. 
As the transition strength $\alpha$ is increased, the efficiency of fluid kinetic energy production decreases below expectation. For deflagrations, this is associated with reduced wall speeds for expanding bubbles and reheating of the region in front of the walls, reducing the pressure difference $[37,48,49]$. The kinetic energy loss leads to a suppression in the gravitational wave power, by a factor which can be as small as $\mathrm{O}\left(10^{-3}\right)$. This means that current models substantially overestimate gravitational wave production from strong transitions with deflagrations. Detonations are less affected.

We model the phase transition with a real scalar field $\phi$, coupled to a perfect fluid. We assume that there is no extra physics generating a magnetic field either before or during the phase transition. The model follows that used in previous work $[31,32,50]$, differing by a change in the effective potential and therefore the equation of state. Our previous work used the high-temperature expansion of the one-loop thermal effective potential, and we found that in stronger transitions, the total energy could drop below the scalar potential energy, which is unphysical. In this scenario, our algorithm would compute the temperature to be imaginary, causing a crash. Indeed, the high-temperature expansion is known to fail well below $T_{\mathrm{c}}$; for example, the speed of sound diverges and then becomes imaginary. To fix this we have introduced a simpler bag model equation of state, described below. The new equation of state changes only how the relevant thermodynamic parameters $\alpha$ and $v_{\mathrm{w}}$ are realized in terms of the parameters of the potential and field-fluid coupling term. The flows around the expanding bubbles, and hence the gravitational wave spectrum, depend on the underlying theory only through $\alpha$ and the wall speed $v_{\mathrm{w}}$, with the overall frequency scale set by the redshifted mean bubble separation.

Our coupled field-fluid system has energy-momentum tensor

$$
T^{\mu \nu}=\partial^{\mu} \phi \partial^{\nu} \phi-\frac{1}{2} g^{\mu \nu}(\partial \phi)^{2}+(\epsilon+p) U^{\mu} U^{\nu}+g^{\mu \nu} p
$$

where $U=\gamma(1, \mathbf{v})$, with fluid 3-velocity $\mathbf{v}$ and associated Lorentz factor $\gamma$. The internal energy $\epsilon$ and pressure $p$ are

$\epsilon=3 a(\phi) T^{4}+V_{0}(\phi), \quad p=a(\phi) T^{4}-V_{0}(\phi)$,

and the enthalpy is $w=\epsilon+p$.

The zero-temperature effective potential is

$$
V_{0}(\phi)=\frac{1}{2} M^{2} \phi^{2}-\frac{1}{3} \mu \phi^{3}+\frac{1}{4} \lambda \phi^{4}-V_{\mathrm{c}},
$$

where $V_{\mathrm{c}}$ is chosen such that $V_{0}\left(\phi_{\mathrm{b}}\right)=0$, and $\phi_{\mathrm{b}}$ is the value of $\phi$ in the broken phase at $T=0$. We denote the potential energy difference between the vacua by $\Delta V_{0}=$ $V_{0}(0)-V_{0}\left(\phi_{\mathrm{b}}\right)$.
We write the thermal effective potential of our bag model as

$$
V(\phi, T)=V_{0}(\phi)-T^{4}\left(a(\phi)-a_{0}\right),
$$

where $a(\phi)$ models the change in degrees of freedom during the transition. We take

$$
a(\phi)=a_{0}-\frac{\Delta V_{0}}{T_{\mathrm{c}}^{4}}\left[3\left(\frac{\phi}{\phi_{\mathrm{b}}}\right)^{2}-2\left(\frac{\phi}{\phi_{\mathrm{b}}}\right)^{3}\right],
$$

where $a_{0}=\left(\pi^{2} / 90\right) g_{*}$ with $g_{*}$ the effective number of relativistic degrees of freedom in the symmetric phase. Both $\phi=0$ and $\phi=\phi_{\mathrm{b}}$ are stationary points of the function for all $T$. For our choice of $a(\phi)$ the minima of $V$ become degenerate at $T=T_{\mathrm{c}}$, as required.

The energy-momentum tensor can be decomposed into field and fluid parts, coupled through a friction term,

$$
\partial_{\mu} T_{\phi}^{\mu \nu}=-\partial_{\mu} T_{\mathrm{f}}^{\mu \nu}=\eta U^{\mu} \partial_{\mu} \phi \partial^{\nu} \phi .
$$

The authors of Ref. [32] used a field- and temperaturedependent friction parameter $\eta=\tilde{\eta} \phi^{2} / T$. Although this models high temperature physics more accurately [51], strong transitions can reach small temperatures and again the high-temperature approximation fails. With small temperatures we also find numerical instabilities and so revert to using a constant $\eta$.

The phase transition strength is parametrized by the trace anomaly difference

$$
\Delta \theta(T)=\frac{1}{4} \frac{d}{d T} \Delta V-\Delta V,
$$

where $\Delta V=V(0, T)-V\left(\phi_{\mathrm{b}}, T\right)$. The strength parameter is then

$$
\alpha=\Delta \theta\left(T_{\mathrm{n}}\right) / \epsilon_{\mathrm{r}}\left(T_{\mathrm{n}}\right),
$$

where $T_{\mathrm{n}}$ is the nucleation temperature and $\epsilon_{\mathrm{r}}=3 w / 4$ the radiation energy density.

We assume that the duration of the phase transition is much less than the Hubble time $H_{\mathrm{n}}^{-1}$, and neglect the effect of expansion. This is comparable to the statement that $H_{\mathrm{n}} R_{*} \ll 1$, where $R_{*}$ is the mean bubble separation. In this regime the contribution of bubble collisions to the gravitational wave signal is negligible. To neglect expansion the final simulation time $t_{\text {fin }}$ must also be much smaller than $H_{\mathrm{n}}^{-1}$. For all our simulations $t_{\text {fin }} \leq 10 R_{*}$.

The mean gravitational wave energy density is

$$
\rho_{\mathrm{gw}}=\frac{1}{32 \pi G} \frac{1}{\mathcal{V}} \int_{\mathcal{V}} \mathrm{d}^{3} x \overline{\hat{h}_{i j}^{\mathrm{TT}} \dot{h}_{i j}^{\mathrm{TT}}},
$$

where $\mathcal{V}$ is the simulation volume, $h_{i j}^{\mathrm{TT}}$ is the transverse traceless metric perturbation, and the line indicates 
averaging over a characteristic period of the gravitational waves. We find $h_{i j}^{\mathrm{TT}}$ in Fourier space by a standard technique $[29,31,52]$, sourced only by the fluid, the dominant contribution when $\alpha \lesssim 1$ and $H_{\mathrm{n}} R_{*} \ll 1[29,31,32]$.

We express the gravitational wave energy density in terms of the parameter $\Omega_{\mathrm{gw}}=\rho_{\mathrm{gw}} / \rho_{\mathrm{c}}$, with $\rho_{\mathrm{c}}$ the critical energy density. Our assumptions on $\alpha$ and $H_{\mathrm{n}} R_{*}$ ensure that $\Omega_{\mathrm{gw}} \ll 1$ at all times. They also ensure that the gravitational backreaction is negligible compared to the pressure forces, as the wavelength of the density perturbations $\sim R_{*}$ is much less than the Jeans length $\sim c_{\mathrm{s}} / H_{\mathrm{n}} \sqrt{\delta}$, where $\delta$ is the energy density contrast averaged over the wavelength being considered (for the parameter space we consider, we determined that the variation of the energy density is at most a factor of 20 in the asymptotic fluid profile that develops around an expanding bubble; $\delta$ will be less than this due to averaging over a given wavelength). We leave a deeper analysis of gravitational backreaction in the case $H_{\mathrm{n}} R_{*} \sim c_{\mathrm{s}} / \sqrt{\delta}$ to a later study.

We perform a series of three-dimensional simulations of the field-fluid system. The simulation code is the same as that used in Ref. [32] except for the above changes.

We scan over $\alpha$ for three subsonic deflagrations with asymptotic wall speeds $v_{\mathrm{w}}=\{0.24,0.44,0.56\}$, and two detonations with $v_{\mathrm{w}}=\{0.82,0.92\}$. The asymptotic wall speeds, and their fluid profiles, are found with a spherically symmetric version of the code $[31,32,53,54]$, run with the same parameters until $t=10000 T_{\mathrm{c}}^{-1}$. As we increase $\alpha$, the maximum velocity of the asymptotic fluid profile $v_{\mathrm{p}}$ increases. For each $v_{\mathrm{w}}$, there is a maximum $v_{\mathrm{p}}$, and hence a maximum strength $\alpha_{\max }$, above which solutions either do not exist (subsonic deflagrations), or change into hybrids. We do not consider hybrids here.

The values of $\eta$ needed for these wall speeds are given in the Supplemental Material [55]. By comparison, the standard model estimate is $\eta \simeq 3 \phi_{\mathrm{b}}^{2} / T_{\mathrm{c}}$ [51,56,57].

All simulations have the number of bubbles $N_{\mathrm{b}}=8$, lattice spacing $\delta x=1.0 T_{\mathrm{c}}^{-1}$, time step $\delta t=0.2 T_{\mathrm{c}}^{-1}$, and $L^{3}=960^{3}$ lattice sites, giving a mean bubble separation $R_{*}=L \delta x / N_{\mathrm{b}}^{1 / 3}=480 T_{\mathrm{c}}^{-1}$. All bubbles are nucleated simultaneously with a Gaussian profile at the same locations at the start of each simulation. The initial profile of the bubbles is insignificant as they approach the same asymptotic profile.

We fix $g_{*}=106.75, M^{2}=0.0427 T_{\mathrm{c}}^{2}, \mu=0.168 T_{\mathrm{c}}$ and $\lambda=0.0732$, in turn fixing $\phi_{\mathrm{b}}=2.0 T_{\mathrm{c}}$. This sets the relative change in degrees of freedom to $\left[a\left(\phi_{\mathrm{b}}\right)-a_{0}\right] / a_{0}=5.9 \times 10^{-3}$. To change the transition strength we vary $T_{\mathrm{n}}$.

We output slices of the temperature $T$, fluid speed $v$, and vorticity magnitude $|\nabla \times \mathbf{v}|$. Movies created from these slices are available at [58]. Selected stills are included in the Supplemental Material [55].

We measure the rms fluid 3-velocity $\bar{v}$, and its irrotational and rotational parts $\bar{v}_{\|}$and $\bar{v}_{\perp}$. We also track the enthalpy-weighted rms four-velocity $\bar{U}_{\mathrm{f}}$ defined as

$$
\bar{U}_{\mathrm{f}}^{2}=\frac{1}{\bar{w} \mathcal{V}} \int_{\mathcal{V}} \mathrm{d}^{3} x w \gamma^{2} v^{2}
$$

where $\bar{w}$ is the mean enthalpy density. This gives an indication of the magnitude of the shear stress, the source of gravitational waves.

A similar quantity $\bar{U}_{\phi}$ can be constructed to track the progress of the phase transition

$$
\bar{U}_{\phi}^{2}=\frac{1}{\bar{w} \mathcal{V}} \int_{\mathcal{V}} \mathrm{d}^{3} x \partial_{i} \phi \partial_{i} \phi
$$

proportional to the total area of the phase boundary. We call the time when $\bar{U}_{\phi}$ reaches its maximum the peak collision time, $t_{\mathrm{pc}}$. Note that $t_{\mathrm{pc}} \propto R_{*} / v_{\mathrm{w}}$. To see how these global quantities evolve during a detonation and a deflagration see Fig. 6 in the Supplemental Material [55].

To check the dependence of our key observables on lattice spacing, we perform simulations with the same physical volume and various lattice spacings $\delta x T_{\mathrm{c}}=$ $\{2.0,1.5,1.0,0.75,0.5\}$ for $v_{\mathrm{w}}=0.24$ and $v_{\mathrm{w}}=0.92$ and $\alpha=0.5$. We find that $\bar{v}_{\perp, \max }^{2}, \bar{U}_{\mathrm{f}, \text { max }}$, and $\Omega_{\mathrm{gw}}$ converge with lattice spacing. We perform a quadratic fit with $\delta x$ for each quantity, finding that $\bar{U}_{\mathrm{f}, \max }$ and $\Omega_{\mathrm{gw}}$ differ from the continuum limit by $\mathrm{O}(1 \%)$. The quantity that is most sensitive to the grid is $\bar{v}_{\perp \text {,max }}^{2}$ which we underestimate from the continuum limit by $\simeq 25 \%$. We also test convergence of key observables with time step, finding in all cases that convergence is better for $\delta t$ than for $\delta x$. It is important to check how close the colliding bubbles are to their asymptotic profile. We find that spherically symmetric bubbles with diameter $R_{\mathrm{c}}$ have at worst $\bar{U}_{\mathrm{f}}$ within $20 \%$ of the asymptotic $\bar{U}_{\mathrm{f}}$. In the Supplemental Material [55] we show our convergence test results and the deviation from asymptotic $\bar{U}_{\mathrm{f}}$ for all $v_{\mathrm{w}}$ and $\alpha$ considered here.

From our simulations we see that a rotational component of velocity is generated during the bubble collision phase. In order to gauge the relative amount of kinetic energy in the rotational component of velocity, we consider the ratio of the maxima of mean square 3-velocities $\bar{v}_{\perp \text { max }}^{2} / \bar{v}_{\text {max }}^{2}$. We plot this in Fig. 1. As we increase $\alpha$ for the deflagrations, we see that the proportion of the velocity found in rotational modes increases dramatically, whereas for detonations it stays constant. The deflagrations with smaller wall velocities have a larger proportion of the velocity in rotational modes. For $v_{\mathrm{w}}=0.24, \alpha=0.34$ the ratio $\bar{v}_{\perp, \max }^{2} / \bar{v}_{\max }^{2}=0.65$, and if we naively extrapolate the trend in the last few points up to $\alpha_{\max }$ this increases to 0.95 .

Figure 7 of the Supplemental Material [55] shows that the vorticity is generated inside the bubbles, not outside where the fluid shells first interact.

To better understand transfer of energy from the scalar field to the fluid, we plot how $\bar{U}_{\phi}$ and $\bar{U}_{\mathrm{f}}$ change as we increase $\alpha$ for detonations with $v_{\mathrm{w}}=0.92$ and deflagrations 


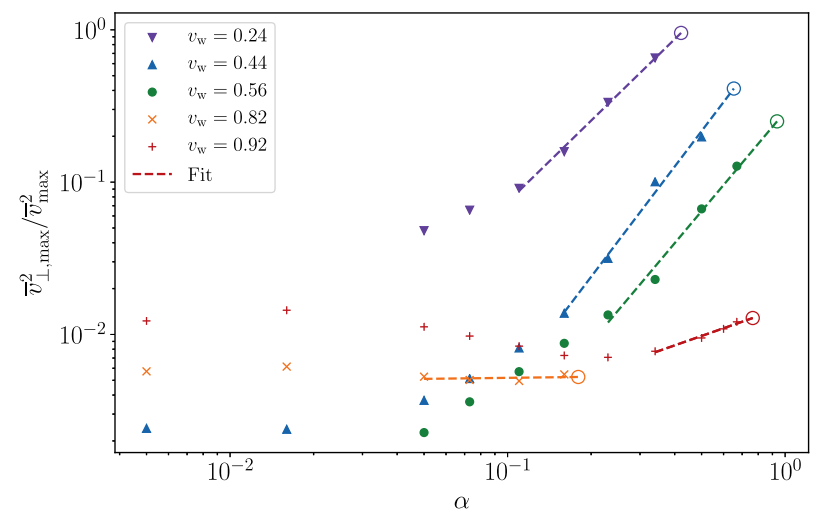

FIG. 1. Proportion of mean square fluid velocity in the rotational modes. We plot the ratio of $\bar{v}_{\perp, \max }$ to $\bar{v}_{\max }$ against $\alpha$. Dashed lines give a linear fit for the last four simulation points. The fits are extrapolated to $\alpha_{\max }$ for deflagrations, or to the largest $\alpha$ for which a wall speed corresponds to a detonation (hollow circles).

with $v_{\text {w }}=0.44$ (Fig. 2). When $\bar{U}_{\phi}$ reaches its maximum, the volumes in each phase are approximately equal. As the phase boundary sweeps out the remaining regions of metastable phase, $\bar{U}_{\phi}$ relaxes to zero. It is striking that for deflagrations the relaxation takes longer as we increase $\alpha$, whereas for detonations the shape of $\bar{U}_{\phi}$ remains unchanged. The phase boundaries in a deflagration must therefore move more slowly in the later stages, as the transition strength increases.

The reason for the slowing is that the metastable phase is reheated by the fluid shells in front of the bubble walls $[37,48,49]$. Towards the end of the transition the remaining metastable phase forms into hot droplets (see Fig. 7 in the Supplemental Material [55]). The higher pressure inside the droplets opposes their collapse.

For detonations, where the fluid shell develops behind the bubble wall, shrinking regions of the metastable phase are not reheated (see Fig. 8 in the Supplemental Material [55]).

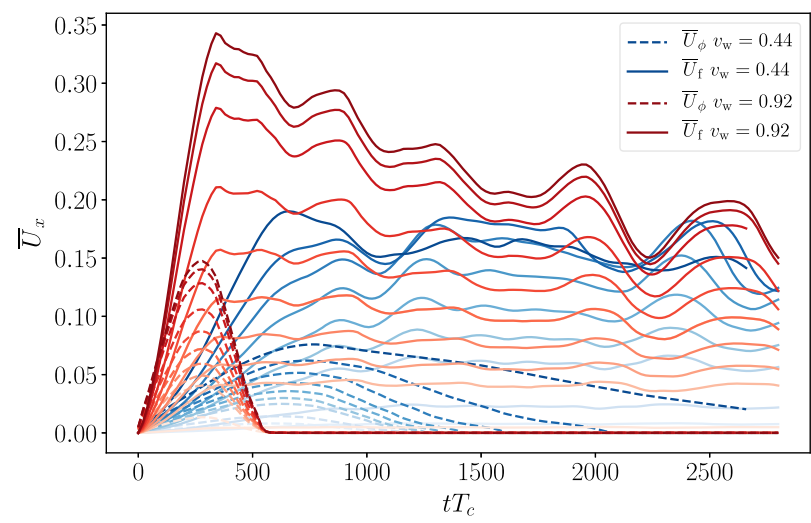

FIG. 2. Evolution of $\bar{U}_{\phi}$ (dashed lines) and $\bar{U}_{\mathrm{f}}$ (solid lines) for simulations with increasing $\alpha$ (darker shades). In blue we show deflagrations with $v_{\mathrm{w}}=0.44$ whereas red lines show detonations with $v_{\mathrm{w}}=0.92$.
Figure 2 also shows that $\bar{U}_{\mathrm{f}}$ increases with $\alpha$, as one expects from the increasing scalar potential energy. However, the maximum is below that expected from a single bubble, which is a good estimate of $\bar{U}_{\mathrm{f}}$ at low $\alpha[31,32]$.

To obtain the single-bubble estimate, simulations of expanding spherical bubbles are performed, and the expected enthalpy-weighted rms velocity $\bar{U}_{f \text {,exp }}$ is that of the fluid shell when the wall reaches a diameter of $R_{*}$. We then take the ratio with the maximum of $\bar{U}_{\mathrm{f}}$ in each simulation, shown in Fig. 3. Note that due to finite volume effects $\bar{U}_{\mathrm{f}}$ oscillates in our simulations, giving an $\mathrm{O}(10 \%)$ uncertainty to this estimate.

For all wall speeds, the ratio of $\bar{U}_{\text {f,max }}$ to $\bar{U}_{f \text {,exp }}$ decreases as we increase the transition strength. However, for deflagrations the decrease in the kinetic efficiency is more dramatic, and more rapid for slower walls: in the slowest deflagration $\left(v_{\mathrm{w}}=0.24\right), \bar{U}_{\mathrm{f}, \max } / \bar{U}_{f, \exp }$ reaches 0.3 . The decrease is approximately linear; a naive linear extrapolation to the maximum possible strength is indicated by open circles. The loss of kinetic energy is probably a result of the slowing discussed above, limiting the transfer of energy.

The deficit in kinetic energy can be expected to reduce the gravitational wave signal. In current modeling $[32,60]$, the expected gravitational wave density parameter from a flow with $\bar{U}_{f \text {,exp }}$ at time $t \ll H_{\mathrm{n}}^{-1}$ is

$$
\Omega_{\mathrm{gw}, \exp }=3 \tilde{\Omega}_{\mathrm{gw}}\left(\frac{\bar{w}}{\bar{\epsilon}}\right)^{2} \bar{U}_{f, \exp }^{4}\left(H_{\mathrm{n}} t\right)\left(H_{\mathrm{n}} R_{*}\right),
$$

where $\tilde{\Omega}_{\mathrm{gw}}$ has been shown to be a constant of $\mathrm{O}\left(10^{-2}\right)$ in weak and intermediate transitions. Here, we take $\tilde{\Omega}_{\mathrm{gw}}=10^{-2}$. In Fig. 4 we plot the ratio of $\Omega_{\mathrm{gw}} / t$ to $\Omega_{\mathrm{gw}, \text { exp }} / t$, where $\Omega_{\mathrm{gw}} / t$ is averaged over the final $\Delta t=$ $2 R_{*}$ of the simulation. In the most extreme case, $v_{\mathrm{w}}=0.24$ and $\alpha=0.34$, the ratio is $2 \times 10^{-3}$. This is even less

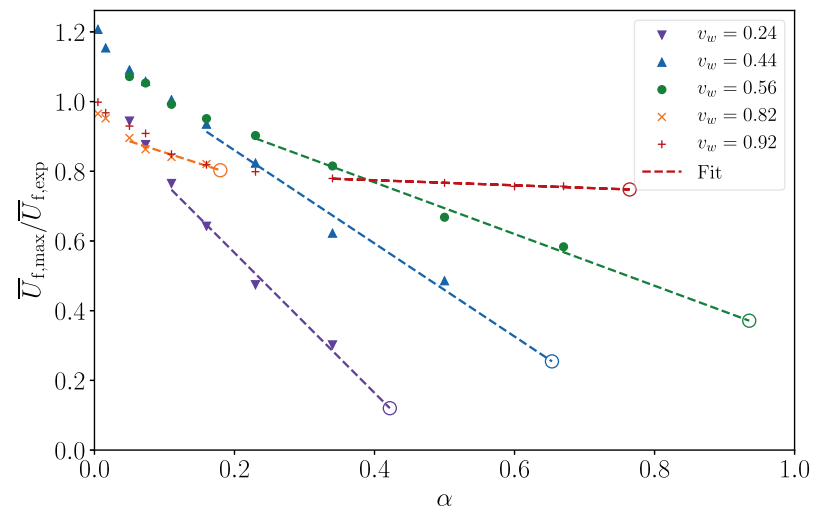

FIG. 3. Comparison between the maximum value of $\bar{U}_{\mathrm{f}}$ in each simulation and that predicted by [59] for the given $v_{\mathrm{w}}$ and $\alpha$. Dashed lines give a linear fit for the last four simulation points. Hollow circles show the extrapolation to $\alpha_{\max }$ for deflagrations, or up to the largest $\alpha$ for which the wall speed corresponds to a detonation. 


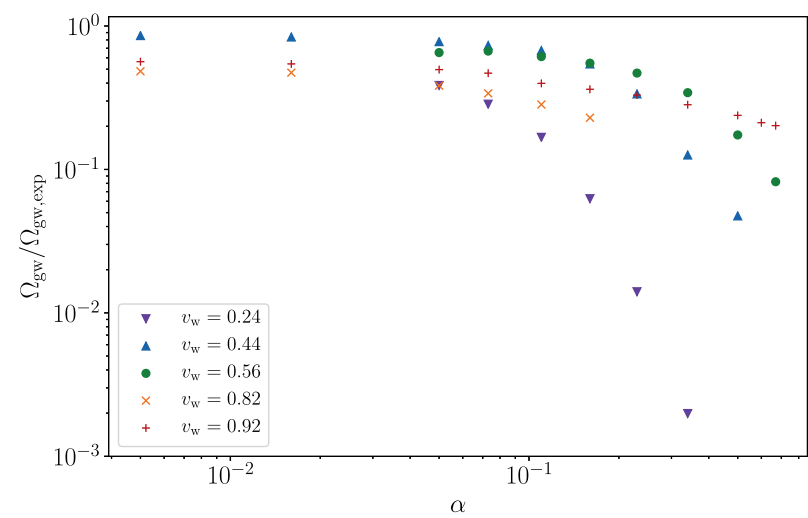

FIG. 4. Comparison of the gravitational waves produced in our simulations against that predicted by Eq. (12) using $\bar{U}_{f \text {,exp }}$ found from $v_{\mathrm{w}}$ and $\alpha$.

than the kinetic energy suppression suggests, a factor of $\left(\bar{U}_{\mathrm{f}, \max } / \bar{U}_{f, \exp }\right)^{4} \simeq 8 \times 10^{-3}$.

A table of simulation parameters and measured quantities can be found in the Supplemental Material [55].

We have performed the first three-dimensional simulations of strong first-order phase transitions, with the strength parameter $\alpha$ up to an order of magnitude larger than those previously studied [32].

A rotational component of velocity $\bar{v}_{\perp}$ is generated during the collision phase. For deflagrations, the ratio $\bar{v}_{\perp, \max }^{2} / \bar{v}_{\max }^{2}$ grows rapidly with $\alpha$, reaching 0.65 for $v_{\mathrm{w}}=0.24$. For detonations, the ratio is $\mathrm{O}\left(10^{-2}\right)$ - showing no consistent trend with $\alpha$.

For stronger phase transitions a smaller proportion of the scalar potential energy is transferred into fluid kinetic energy than is expected from the behaviour of isolated bubbles. For deflagrations, we suppose that the deficit is due to reheating of the metastable phase slowing the bubble walls during the collision phase. The deficit can be substantial, with $\bar{U}_{\mathrm{f}, \max } / \bar{U}_{f \text {,exp }}$ falling to $\sim 0.3$ for $v_{\mathrm{w}}=$ 0.24 in our simulations, and could fall as low as 0.1 using a naive linear extrapolation to the maximum possible strength at that wall speed.

The gravitational wave intensity is lower than expected, by a factor of order $10^{-3}$ for the strongest deflagration with the lowest wall speed. This can mostly be accounted for by the kinetic energy deficit. Detonations do not suffer such a dramatic suppression, with the smallest suppression factor about 0.2 for $v_{\mathrm{w}}=0.92$.

Our results have important consequences for gravitational waves from phase transitions. They indicate that the current model $[32,60]$ overestimates the gravitational wave power spectrum for strong transitions, by a factor of a few for detonations, and by an order of magnitude or more for deflagrations. We estimate (We use PTPlot [61], to compute and plot signal-to-noise ratio curves from first-order phase transitions for LISA. We choose $g_{*}=106.75$ and an optimistic $T_{\mathrm{n}}=100 \mathrm{GeV}$. The resulting plot shows that a signal-to-noise ratio of 10 requires $\bar{U}_{\mathrm{f}}$ of at least 0.07 for all $H_{\mathrm{n}} R_{*}$.) that to obtain a signal-to-noise ratio of $10 \bar{U}_{\mathrm{f}}$ must be at least 0.07. Therefore the kinetic energy suppression we observe will probably render transitions with $v_{\mathrm{w}}=0.24$ unobservable except for within a very small region of parameter space. Faster walls suffer less suppression, though the observable parameter space is still reduced.

We plan larger simulations to characterize more precisely the suppression, and its effect on observability.

The authors would like to thank Chiara Caprini, Kari Rummukainen, and Danièle Steer for helpful discussions. Our simulations made use of the resources of the Finnish Centre for Scientific Computing CSC. D. C. is supported by an STFC Studentship. M. H. acknowledges support from the Science and Technology Facilities Council, Grant No. ST/P000819/1. D. J. W. is supported by a Science and Technology Facilities Council Ernest Rutherford Fellowship, Grant No. ST/R003904/1, by the Research Funds of the University of Helsinki, and by the Academy of Finland, Grant No. 286769.

*d.cutting@sussex.ac.uk †m.b.hindmarsh@sussex.ac.uk +david.weir@nottingham.ac.uk

[1] H. Audley et al., arXiv:1702.00786.

[2] K. Kajantie, M. Laine, K. Rummukainen, and M. E. Shaposhnikov, Phys. Rev. Lett. 77, 2887 (1996).

[3] K. Kajantie, M. Laine, K. Rummukainen, and M. E. Shaposhnikov, Nucl. Phys. B493, 413 (1997).

[4] S. Profumo, M. J. Ramsey-Musolf, and G. Shaughnessy, J. High Energy Phys. 08 (2007) 010.

[5] J. R. Espinosa, T. Konstandin, and F. Riva, Nucl. Phys. B854, 592 (2012).

[6] J. M. Cline and K. Kainulainen, J. Cosmol. Astropart. Phys. 01 (2013) 012.

[7] S. Profumo, M. J. Ramsey-Musolf, C. L. Wainwright, and P. Winslow, Phys. Rev. D 91, 035018 (2015).

[8] A. Beniwal, M. Lewicki, M. White, and A. G. Williams, J. High Energy Phys. 02 (2019) 183.

[9] M. Kakizaki, S. Kanemura, and T. Matsui, Phys. Rev. D 92, 115007 (2015).

[10] G. C. Dorsch, S. J. Huber, T. Konstandin, and J. M. No, J. Cosmol. Astropart. Phys. 05 (2017) 052.

[11] P. Basler, M. Krause, M. Muhlleitner, J. Wittbrodt, and A. Wlotzka, J. High Energy Phys. 02 (2017) 121.

[12] L. Randall and G. Servant, J. High Energy Phys. 05 (2007) 054.

[13] T. Konstandin, G. Nardini, and M. Quiros, Phys. Rev. D 82, 083513 (2010).

[14] T. Konstandin and G. Servant, J. Cosmol. Astropart. Phys. 12 (2011) 009.

[15] B. von Harling and G. Servant, J. High Energy Phys. 01 (2018) 159.

[16] B. M. Dillon, B. K. El-Menoufi, S. J. Huber, and J. P. Manuel, Phys. Rev. D 98, 086005 (2018). 
[17] E. Megías, G. Nardini, and M. Quirós, J. High Energy Phys. 09 (2018) 095.

[18] S. Bruggisser, B. Von Harling, O. Matsedonskyi, and G. Servant, J. High Energy Phys. 12 (2018) 099.

[19] P. Schwaller, Phys. Rev. Lett. 115, 181101 (2015).

[20] A. Addazi and A. Marciano, Chin. Phys. C 42, 023107 (2018).

[21] M. Aoki, H. Goto, and J. Kubo, Phys. Rev. D 96, 075045 (2017).

[22] D. Croon, V. Sanz, and G. White, J. High Energy Phys. 08 (2018) 203.

[23] M. Breitbach, J. Kopp, E. Madge, T. Opferkuch, and P. Schwaller, J. Cosmol. Astropart. Phys. 07 (2019) 007.

[24] N. Okada and O. Seto, Phys. Rev. D 98, 063532 (2018).

[25] T. Hasegawa, N. Okada, and O. Seto, Phys. Rev. D 99, 095039 (2019).

[26] T. Gorda, A. Helset, L. Niemi, T. V. I. Tenkanen, and D. J. Weir, J. High Energy Phys. 02 (2019) 081.

[27] O. Gould, J. Kozaczuk, L. Niemi, M. J. Ramsey-Musolf, T. V. I. Tenkanen, and D. J. Weir, Phys. Rev. D 100, 115024 (2019).

[28] K. Kainulainen, V. Keus, L. Niemi, K. Rummukainen, T. V. I. Tenkanen, and V. Vaskonen, J. High Energy Phys. 06 (2019) 075.

[29] M. Hindmarsh, S. J. Huber, K. Rummukainen, and D. J. Weir, Phys. Rev. Lett. 112, 041301 (2014).

[30] J. T. Giblin and J. B. Mertens, Phys. Rev. D 90, 023532 (2014).

[31] M. Hindmarsh, S. J. Huber, K. Rummukainen, and D. J. Weir, Phys. Rev. D 92, 123009 (2015).

[32] M. Hindmarsh, S. J. Huber, K. Rummukainen, and D. J. Weir, Phys. Rev. D 96, 103520 (2017).

[33] M. Hindmarsh, Phys. Rev. Lett. 120, 071301 (2018).

[34] R. Jinno and M. Takimoto, Phys. Rev. D 95, 024009 (2017).

[35] T. Konstandin, J. Cosmol. Astropart. Phys. 03 (2018) 047.

[36] E. Witten, Phys. Rev. D 30, 272 (1984).

[37] H. Kurki-Suonio, Nucl. Phys. B255, 231 (1985).

[38] M. Kamionkowski, A. Kosowsky, and M. S. Turner, Phys. Rev. D 49, 2837 (1994).

[39] C. Caprini, R. Durrer, and G. Servant, Phys. Rev. D 77, 124015 (2008).

[40] G. Gogoberidze, T. Kahniashvili, and A. Kosowsky, Phys. Rev. D 76, 083002 (2007).
[41] C. Caprini, R. Durrer, and G. Servant, J. Cosmol. Astropart. Phys. 12 (2009) 024.

[42] C. Caprini, R. Durrer, T. Konstandin, and G. Servant, Phys. Rev. D 79, 083519 (2009).

[43] P. Niksa, M. Schlederer, and G. Sigl, Classical Quantum Gravity 35, 144001 (2018).

[44] J. Ellis, M. Lewicki, and J. M. No, J. Cosmol. Astropart. Phys. 04 (2019) 003.

[45] J. Ellis, M. Lewicki, J. M. No, and V. Vaskonen, J. Cosmol. Astropart. Phys. 06 (2019) 024.

[46] A. R. Pol, S. Mandal, A. Brandenburg, T. Kahniashvili, and A. Kosowsky, arXiv:1903.08585.

[47] R. Jinno, H. Seong, M. Takimoto, and C. M. Um, J. Cosmol. Astropart. Phys. 10 (2019) 033.

[48] T. Konstandin and J. M. No, J. Cosmol. Astropart. Phys. 02 (2011) 008.

[49] A. Mégevand and S. Ramírez, Nucl. Phys. B928, 38 (2018).

[50] J. Ignatius, K. Kajantie, H. Kurki-Suonio, and M. Laine, Phys. Rev. D 49, 3854 (1994).

[51] B.-H. Liu, L. D. McLerran, and N. Turok, Phys. Rev. D 46, 2668 (1992).

[52] J. Garcia-Bellido, D. G. Figueroa, and A. Sastre, Phys. Rev. D 77, 043517 (2008).

[53] H. Kurki-Suonio and M. Laine, Phys. Rev. D 54, 7163 (1996).

[54] H. Kurki-Suonio and M. Laine, Phys. Rev. D 51, 5431 (1995).

[55] See Supplemental Material at http://link.aps.org/ supplemental/10.1103/PhysRevLett.125.021302 for more discussion on the equations of motion, resolution convergence, supplementary plots, and simulation stills.

[56] P. John and M. G. Schmidt, Nucl. Phys. B598, 291 (2001); B648, 449(E) (2003).

[57] G. D. Moore and T. Prokopec, Phys. Rev. D 52, 7182 (1995).

[58] D. Cutting, movies of strong phase transitions, https://vimeo .com/album/5968055 (2019).

[59] J. R. Espinosa, T. Konstandin, J. M. No, and G. Servant, J. Cosmol. Astropart. Phys. 06 (2010) 028.

[60] C. Caprini et al., J. Cosmol. Astropart. Phys. 04 (2016) 001.

[61] C. Caprini et al., J. Cosmol. Astropart. Phys. 03 (2020) 024; PTPlot v1.01, http://www.ptplot.org/ptplot/. 